\title{
Hawking radiation and conformal anomalies in two-dimensional AdS spacetimes
}

\author{
Daniel A. Drehmer*广 and Vilson T. Zanchin ${ }^{\dagger}$ \\ Centro de Ciências Naturais e Humanas - CCNH, Universidade Federal do ABC - UFABC \\ Rua Santa Adélia, 166, 09210-170, Santo André, Brazil \\ E-mail: daniel.drehmer@ufabc.edu.br, \\ zanchin@ufabc.edu.br
}

\begin{abstract}
The Hawking radiation is one of most interesting known effects of black hole physics. Since its discovery in 1974 by Hawking, this phenomenon has been an object of study of great interest. Despite the large number of works on this subject, details of the quantum evolution of black holes are still unknown. In large part, this is due to the complexity of the calculations necessary to get such results. In order to calculate the Hawking radiation, but avoiding mathematical complications, many years ago Christensen and Fulling had proposed a method to derive the Hawking radiation flux in terms of trace anomalies, which is a very simple way to obtain consistent results, since the anomaly is given in terms of the spacetime curvature invariants. This approach is successful in the calculation of the stress tensor of the Hawking radiation emitted by black holes in two-dimensional spacetimes, where all the nonzero components of the stress tensor can be obtained. However, in the case of four-dimensional spacetimes the Christensen-Fulling method does not furnish a complete result, since the transverse components of the stress tensor cannot be determined. In this work we review such an approach and apply it to two-dimensional static black holes in asymptotically Minkowski and anti-de Sitter (AdS) spacetimes. For black holes in asymptotically AdS spacetimes a straightforward application of the Christensen-Fulling method does not provide correct results. To overcome this problem, we propose a slight modification of the method, where we argue that the trace anomaly due the background curvature of the AdS spacetime, without the black hole, should be deducted from the total trace anomaly, and hence we get the correct results for the energy flux of the Hawking radiation in all AdS spacetimes we have tested.
\end{abstract}

5th International School on Field Theory and Gravitation,

April 20 - 242009

Cuiabá city, Brazil

\footnotetext{
*Poster section

${ }^{\dagger}$ We thank Conselho Nacional de Desenvolvimento Científico e Tecnológico - CNPq, Brazil, and Coordenação de Aperfeiçoamento de Pessoal de Nível Superior - CAPES, Brazil, for scholarships.
} 


\section{Hawking radiation and the Christensen-Fulling approach}

According to classical physics it is expected that nothing can leave the interior of a black hole. That is, any particle that crosses the black hole horizon is subject to a so strong gravitational field that it can never return, and so, being ordered to fall into the physical singularity inside such objects. As shown by Hawking [1, 2], this scenario is modified when quantum effects are considered in the vicinity of a black hole, or in an accelerated reference frame. In both of these cases, there is particle creation. Hawking showed that a black hole can evaporate, emitting radiation with a characteristic black body spectrum, with temperature entirely determined by its mass, electric charge and angular momentum. This radiation emission could cause a total "disappearance" of the black hole, making it expel all its mass in a large explosion.

In the method proposed by Christensen and Fulling [3], the trace anomaly is used to obtain the flux of energy due to Hawking radiation. In such an approach it is assumed that the quantum states preserve the symmetry of the spacetime, which reflects on the symmetry of the energy-momentum tensor. Moreover, it is assumed that the energy-momentum tensor is time independent. With these hypotheses, the conservation equation

$$
\nabla_{\mu} T_{v}^{\mu}=0
$$

provides equations for all the nonzero components of energy-momentum tensor, except for the energy density $T_{t}^{t}$, which can be obtained through the relation

$$
T_{t}^{t}=T_{\alpha}^{\alpha}-T_{i}^{i}
$$

as long as the trace of the energy-momentum tensor is known. In general, the equations for the nonzero components of the stress tensor provided by (1.1) can be solved in terms of a function of the trace of the anomalous stress tensor and of two integration constants. Such constants can be determined by physically acceptable boundary conditions in the asymptotic limits.

\section{The spacetime geometry}

The geometry of the spacetime in the vicinity of the black holes studied in this work can be described by a metric of the form

$$
d s^{2}=-f(r) d t^{2}+\frac{1}{f(r)} d r^{2}+r^{2} d \Omega^{2}
$$

where $f(r)$ is given by

$$
f(r)=a+\alpha^{2} r^{2}-\frac{b}{r}+\frac{c}{r^{2}} .
$$

The parameters $b$ and $c$ are proportional, respectively, to the mass and to the electric charge squared of the black hole, $\alpha$ is related to the cosmological constant by $\alpha^{2}=-\frac{1}{3} \Lambda$. Parameter $a$ assumes the values $-1,0$ and +1 . The event horizon is given by the largest root $\left(r_{h}\right)$ of the equation $f(r)=0$, such that $f(r)>0$ for $r>r_{h}$. The metric of the surfaces of constant $t$ and $r$ is given by $d \Omega^{2}=d \theta^{2}+Z(\theta)^{2} d \varphi^{2}$, where the function $Z(\theta)$ depends on the value of $a$, and there are three possibilities: (i) Hyperbolic symmetry $(Z(\theta)=\sinh \theta$ ) when $a=-1$; (ii) Planar symmetry $(Z(\theta)=1)$ when $a=0$; Spherical symmetry $(Z(\theta)=\sin \theta)$ when $a=1$ [4]. 
Using techniques of dimensional reduction, it is possible to obtain an equivalent two-dimensional metric given by

$$
d s^{2}=-f(r) d t^{2}+\frac{1}{f(r)} d r^{2}
$$

In this work we focus on this two-dimensional metric.

\section{Trace anomalies}

In quantum field theory an anomaly is a break of a classical symmetry. An important effect of quantization of fields in curved spacetime is the emergence of gravitational anomalies (also called conformal anomalies). Such anomalies arise as a violation of energy conservation and, for instance, in the case of massless fields they appear as the conformal invariance violation. This symmetry break appears explicitly in the energy-momentum tensor, which acquires nonzero trace.

In general, the trace of the anomalous energy-momentum tensor can be given in terms of the spacetime curvature invariants [5]. For a single scalar field evolving in a two-dimensional spacetime the trace anomaly of the stress tensor is given by

$$
T_{\alpha}^{\alpha}=\frac{1}{24 \pi} R
$$

where $R$ stands for the Ricci scalar.

\section{The stress tensor for two-dimensional black holes}

For the two-dimensional metric (2.3) the Ricci scalar is given by, $R=-f^{\prime \prime}(r)$, and thus the trace anomaly is

$$
T_{\alpha}^{\alpha}=-\frac{1}{24 \pi}\left(2 \alpha^{2}-\frac{2 b}{r^{3}}+\frac{6 c}{r^{4}}\right) .
$$

From the conservation equation (1.1) and from the trace identity (1.2) we find that the equations for the nonzero components of the stress tensor are

$$
\partial_{r} T_{t}^{r}=0, \quad \partial_{r} T_{r}^{r}+\frac{f^{\prime}}{2 f}\left(2 T_{r}^{r}-T_{\alpha}^{\alpha}\right)=0,
$$

which integrate respectively to

$$
T_{t}^{r}=-A, \quad T_{r}^{r}(r)=\frac{1}{f(r)}\left\{B-A+H_{2 D}(r)\right\},
$$

where $A$ And $B$ are two integration constants, and the function $H_{2 D}(r)$ is given by

$$
H_{2 D}(r)=\int_{r_{h}}^{r} \frac{f^{\prime}\left(r^{\prime}\right)}{2} T_{\alpha}^{\alpha}\left(r^{\prime}\right) d r^{\prime}
$$

The total energy density, $T_{t}^{t}$, can be obtained using the trace identity (1.2). 
Therefore, in terms of the coordinates $\left(t, r^{*}\right), r^{*}$ being the tortoise coordinate, the full stress tensor can be written in the form

$$
T_{v}^{* \mu}=\left[\begin{array}{cc}
T_{\alpha}{ }^{\alpha}(r)-\frac{H_{2 D}(r)}{f(r)} & 0 \\
0 & \frac{H_{2 D}(r)}{f(r)}
\end{array}\right]+\frac{A}{f(r)}\left[\begin{array}{cc}
1 & -1 \\
1 & -1
\end{array}\right]+\frac{B}{f(r)}\left[\begin{array}{cc}
-1 & 0 \\
0 & 1
\end{array}\right] .
$$

In order to determine the integration constants $A$ and $B$, it is appropriate to write the stress tensor as a function of the null coordinates $u=t+r^{*}$ and $v=t-r^{*}$. Thus, we have

$$
T_{u u}=\frac{1}{4}\left[2 B+2 H_{2}(r)-f(r) T_{\alpha}^{\alpha}(r)\right], \quad T_{u v}=T_{v u}=-\frac{1}{4} f(r) T_{\alpha}^{\alpha}(r), \quad T_{v v}=T_{u u}-A,
$$

The quantum state that represents the Hawking radiation is the vacuum state that satisfies the Unruh vacuum boundary conditions, i.e., no flux of ingoing particles at infinity, $T_{v v}(r \rightarrow \infty)=0$, and without energy flux at the past horizon, $T_{u u}(V \rightarrow 0)=0$. With this conditions the integration constants become

$$
A=\lim _{r \rightarrow \infty}\left[T_{u u}(r)-T_{v v}(r)\right], \quad B=0 .
$$

Hence, for a single scalar field the energy flux of the particles created by the black hole is given be

$$
T_{t}^{r}(\rightarrow \infty)=A=\frac{1}{48 \pi} \kappa^{2}+\frac{1}{48 \pi} \lim _{r \rightarrow \infty}\left(f(r) f^{\prime \prime}(r)-\frac{\left[f^{\prime}(r)\right]^{2}}{4}\right) .
$$

This result holds for all nonrotating black holes in two spacetime dimensions, independently from its energy and mater content. However, in order to determine the integration constants we must specify the global charges, i.e., the values of the parameters $a, b$ and $c$. We perform such an analysis for four distinct black holes in the following.

\subsection{Asymptotically Minkowski spacetimes}

Spacetimes with an asymptotic trivial structure are spacetimes for which the metric becomes the Minkowski metric $g_{\mu \nu}=\operatorname{diag}\{-1,1,1,1\}$ in the asymptotic region $(r \rightarrow \infty)$. For such kind of spacetimes the term in the horizon function $f(r)$, cf. equation (2.2), related to the cosmological constant cannot be present, which means $\Lambda=0$. Next we calculate the energy flux of Hawking radiation for two spherically symmetric black holes in spacetimes with Minkowskian asymptotic structure.

\subsubsection{Schwarzschild black hole}

For the Schwarzschild spacetime the global parameters that define the mass $M$ and electric charge of the black hole in the horizon function (2.2) are respectively $b=2 M$ and $c=0$, i.e., no electric charge. The spherical symmetry is established by $a=1$. In this case, the trace anomaly (3.1) results in $T_{\alpha}{ }^{\alpha}=\frac{M}{6 \pi r^{3}}$, and then, $H_{2 D}(r)=\frac{M^{2}}{24 \pi}\left(\frac{1}{16 M^{4}}-\frac{1}{r^{4}}\right)$. With these results and equations (4.6) and (4.7) we obtain the energy flux of the Hawking Radiation emitted by the Schwarzschild black hole,

$$
T_{t}^{r}(r \rightarrow \infty)=-\frac{1}{768 \pi M^{2}}
$$

This result agrees with the original result obtained by Christensen and Fulling [3]. 


\subsubsection{Reissner-Nordström black hole}

For the Reissner-Nordström spacetime the global parameters are $a=1, b=2 M$ and $c=Q$, in the horizon function (2.2). The trace anomaly becomes $T_{\alpha}{ }^{\alpha}=\frac{1}{24 \pi}\left(\frac{4 M}{r^{3}}-\frac{6 Q^{2}}{r^{4}}\right)$, and hence we have, $H_{2 D}(r)=\frac{1}{24 \pi}\left(-\frac{M^{2}}{r^{4}}+\frac{2 M Q^{2}}{r^{5}}-\frac{Q^{4}}{r^{6}}+\frac{M^{2}}{r_{h}^{4}}-\frac{2 M Q^{2}}{r_{h}^{5}}+\frac{Q^{4}}{r_{h}^{6}}\right)$. The energy flux of the radiation is

$$
T_{t}^{r}(r \rightarrow \infty)=-\frac{M^{2}-Q^{2}}{48 \pi r_{h}^{4}} .
$$

\subsection{Asymptotically anti-de Sitter spacetimes}

Spacetimes with a non-trivial asymptotic structure, i.e., with a nonzero curvature at infinity and for which the curvature is negative are known as asymptotically anti-de Sitter spacetimes. In particular, the metric of of the anti-de Sitter spacetime is given by (2.1) with $f(r)=1+\alpha^{2} r^{2}$. Note that this spacetime does not present a black hole, but have a nonzero trace given by $T_{\alpha}^{\alpha}=-\frac{\alpha^{2}}{12 \pi}$. Following the same procedure as above, we obtain that the components of the stress tensor in terms of null coordinates $u$ and $v$ are $T_{u u}=\frac{\alpha^{2}}{48 \pi}, T_{v v}=\frac{\alpha^{2}}{48 \pi}-A$ and $T_{u v}=T_{v u}=-\frac{1}{4} f(r) T_{\alpha}^{\alpha}(r)=$ $\frac{\alpha^{2}}{48 \pi}\left(1+\alpha^{2} r^{2}\right)$. Thus, we have a nonzero energy density at infinity. Moreover, both the components $T_{u v}$ and $T_{v u}$ diverge at infinity. These nonzero energy density and radiation flux cannot be associated to quantum effects due to a black hole. Let us present two examples of such inconsistency.

\subsubsection{Schwarzschild-anti-de Sitter black hole}

The geometry of the Schwarzschild-AdS spacetime is given by the metric (2.1) and a horizon function (2.2) with global parameters $a=1, b=2 M, c=0$ and with a nonzero cosmological constant $\alpha \neq 0$. For this black hole the Christensen-Fulling approach gives that energy flux (4.8) is

$$
T_{t}^{r}(r)=-\frac{1}{48 \pi}\left[\alpha^{4} r^{2}+2 \alpha^{2}+\frac{M^{2}}{r_{h}^{4}}+\frac{2 M \alpha^{2}}{r_{h}}+\alpha^{4} r_{h}^{2}\right],
$$

which diverges at infinity. Of course, this result does not agree with the energy flux of the Hawking radiation [2].

\subsubsection{Black string}

The charged planar black string [4] has global parameters given by $a=0, b=4 M, c=4 Q^{2}$ and $\alpha \neq 0$, for which we obtain the following energy flux of radiation

$$
T_{t}^{r}(r)=\frac{1}{24 \pi}\left[\alpha^{4} r^{2}+\alpha^{4} r_{h}^{2}+\frac{4 \alpha^{2} M}{r_{h}}-\frac{8 \alpha^{2} Q^{2}}{r_{h}^{2}}+\frac{4 M^{2}}{r_{h}^{4}}-\frac{16 M Q^{2}}{r_{h}^{5}}+\frac{16 Q^{4}}{r_{h}^{6}}\right],
$$

which again does not agree with the Hawking result [2].

\section{Modification of the Christensen-Fulling approach for AdS spacetimes}

We propose here a slight modification of the Christensen-Fulling method for asymptotically AdS spacetimes. We do this by subtracting the trace anomaly of the stress tensor due to the background metric, i.e., we eliminate the anomaly due the curvature of the background AdS 
spacetime, without the black hole: $T_{(b) \alpha}^{\alpha}=-\frac{\alpha^{2}}{12 \pi}$. Thus, we get an effective anomaly given by, $T_{(e f)_{\alpha}^{\alpha}}^{\alpha}=T_{\alpha}^{\alpha}-T_{(b) \alpha}^{\alpha}$, that is due solely to the black hole. For the horizon function (2.2) this effective anomaly results in

$$
T_{(e f) \alpha}^{\alpha}=-\frac{1}{24 \pi}\left(f^{\prime \prime}(r)-f_{(b)}^{\prime \prime}(r)\right) .
$$

Using this effective anomaly to calculate the energy flux of the Hawking radiation we obtain that

$$
A=\frac{1}{48 \pi} \kappa^{2}+\frac{1}{48 \pi} \lim _{r \rightarrow \infty}\left[f(r) f^{\prime \prime}(r)-f_{(b)}(r) f_{(b)}^{\prime \prime}(r)-\frac{1}{4}\left(\left[f^{\prime}(r)\right]^{2}-\left[f_{(b)}^{\prime}(r)\right]^{2}\right)\right] .
$$

It is easy to see that the second term of this equation vanishes, giving the correct result for the Hawking radiation [1,2]. Also, the components, $T_{u v}=T_{v u}=-\frac{1}{4} f(r) T_{(e f)}^{\alpha} \alpha(r)=0$, of the stress tensor are now well defined in the asymptotic limit.

In fact, using the result (5.2) and replacing the values of the global parameters of mass and electric charge we get the correct energy flux of Hawking radiation for the Schwarzschild-AdS black hole, namely,

$$
T_{t}^{r}(r \rightarrow \infty)=-\frac{1}{48 \pi}\left[\alpha^{4} r_{h}^{2}+\frac{2 M \alpha^{2}}{r_{h}}+\frac{M^{2}}{r_{h}^{4}}\right] .
$$

Also, for the black string we obtain

$$
T_{t}^{r}(r \rightarrow \infty)=-\frac{1}{48 \pi}\left[\alpha^{4} r_{h}^{2}+\frac{4 \alpha^{2} M}{r_{h}}+\frac{4 \alpha^{2} Q^{2}}{r_{h}^{2}}+\frac{4 M^{2}}{r_{h}^{4}}-\frac{16 M Q^{2}}{r_{h}^{5}}+\frac{16 Q^{4}}{r_{h}^{6}}\right] .
$$

as expected.

\section{Conclusions}

In this paper we reviewed the Christensen-Fulling method to calculate the stress tensor of the Hawking radiation emitted by black holes. The application of this method is well established for asymptotically Minkowski spacetimes. However, as seen above for spacetimes with nontrivial asymptotic structure this method fails. We present a proposal to circumvent this problem by subtracting the trace anomaly due to the background metric, and show that the resulting effective trace anomaly and the same Christensen-Fulling approach give then the correct energy flux for a few particular cases.

\section{References}

[1] S. W. Hawking, Black hole explosions?, Nature 248, 30 (1974).

[2] S. W. Hawking, Particle Creation by Black Holes, Commun. Math. Phys. 43, 199 (1975).

[3] S. M. Christensen S. A. Fulling, Trace Anomalies and the Hawking Effect, Phys. Rev. D15, 2088 (1977).

[4] P. S. J. Lemos and V. T. Zanchin, Rotating charged black strings and three-dimensional black holes, Phys. Rev. D54, 3840 (1996).

[5] S. Deser, M. J. Duff and C. J. Ishan, Non-local conformal anomalies, Nucl. Phys. B 111, 45 (1976) 